\title{
Photorelease of Alcohols from 2-Nitrobenzyl Ethers Proceeds via Hemiacetals and May Be Further Retarded by Buffers Intercepting the Primary aci-Nitro Intermediates
}

\author{
Bruno Hellrung, Yavor Kamdzhilov, Markus Schwörer, and Jakob Wirz* \\ Departement Chemie der Universität Basel, Klingelbergstrasse 80, CH-4056 Basel, Switzerland
}

\section{SUPPORTING INFORMATION}

Ionic strength of aqueous solutions $I$ was kept at $0.1 \mathrm{M}$ by addition of $\mathrm{NaCl}$. $\mathrm{pH}$ values refer to calculated ${ }^{1}$ proton concentrations. Solutions of $\mathrm{HCl}$ were prepared with volumetric solutions from Riedel-de Haën. The following buffers were used: HAc/NaAc $(0.025$ $\mathrm{M} / 0.005 \mathrm{M}, \mathrm{pH} 3.87 ; 0.01 \mathrm{M} / 0.01 \mathrm{M}, \mathrm{pH} 4.57 ; 0.005 \mathrm{M} / 0.025 \mathrm{M}, \mathrm{pH}$ 5.27) and $\mathrm{KH}_{2} \mathrm{PO}_{4} / \mathrm{Na}_{2} \mathrm{HPO}_{4}(0.025 \mathrm{M} / 0.005 \mathrm{M}, \mathrm{pH} 6.08 ; 0.01$ $\mathrm{M} / 0.01 \mathrm{M}, \mathrm{pH} 6.78 ; 0.005 \mathrm{M} / 0.015 \mathrm{M}, \mathrm{pH} 7.26)$.

The NMR spectra of the metastable intermediate 3 were collected on a Bruker Avance DRX 500-MHz NMR spectrometer with a BBO probe-head that was kept at $5{ }^{\circ} \mathrm{C}$. HMQC (one-bond $\mathrm{C}-\mathrm{H}$ correlations) was optimized for coupling constants of $155 \mathrm{~Hz}, \mathrm{HMBC}$ (longrange $\mathrm{C}-\mathrm{H}$ correlations) for $7 \mathrm{~Hz}$. The signal of acetonitrile was used as a standard $(\delta 1.94 \mathrm{ppm}){ }^{1}{ }^{1} \mathrm{H}-\mathrm{NMR}(500 \mathrm{MHz}, \delta)$ : $\mathrm{H} 6{ }^{\prime}(8.05, \mathrm{dd}, J=$ $1.3,7.8 \mathrm{~Hz}, 1 \mathrm{H}), \mathrm{H}^{\prime}$ ' (7.92, ddd, $\left.J=1.3,7.6,8.0 \mathrm{~Hz}, 1 \mathrm{H}\right), \mathrm{H} 4^{\prime}(7.55$, ddd, $J=1.3,7.4,8.0 \mathrm{~Hz}, 1 \mathrm{H}), \mathrm{H} 3{ }^{\prime}(6.34, \mathrm{dd}, J=1.3,8.2 \mathrm{~Hz}, 1 \mathrm{H}), \mathrm{H} 2$

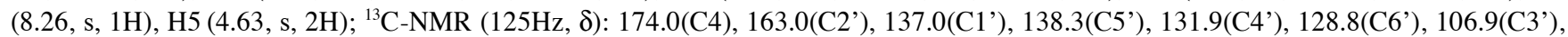

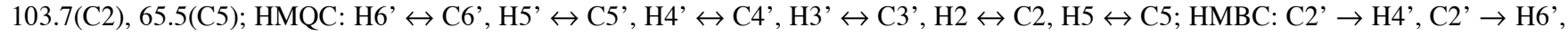
$\mathrm{C} 1^{\prime} \rightarrow \mathrm{H} 2, \mathrm{C}^{\prime}{ }^{\prime} \rightarrow \mathrm{H}^{\prime}, \mathrm{C}^{\prime} \rightarrow \mathrm{H} 2, \mathrm{C} 2 \rightarrow \mathrm{H} 5, \mathrm{C} 4 \rightarrow \mathrm{H} 5$

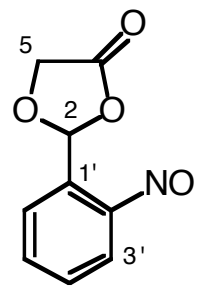

3

Table S1: Average Rate Constants and Standard Deviations for the Rate Constants of Reactions ab1, ab2, bc1, and bc2 in Aqueous Solution ${ }^{a)}$.

\begin{tabular}{|c|c|c|c|c|c|}
\hline$\overline{\mathrm{pH}}$ & {$[\mathrm{HB}] /[\mathrm{B}]$} & $\begin{array}{l}k_{\mathrm{ab} 1} \times 10^{-5} / \mathrm{s}^{-1} \\
\left(\lambda_{\mathrm{obs}} 420 \mathrm{~nm}\right)\end{array}$ & $\begin{array}{l}k_{\mathrm{ab} 2} \times 10^{-4} / \mathrm{s}^{-1} \\
\left(\lambda_{\mathrm{obs}} 420 \mathrm{~nm}\right)\end{array}$ & $\begin{array}{l}k_{\mathrm{bc} 1} \times 10^{-3} / \mathrm{s}^{-1} \\
\left(\lambda_{\mathrm{obs}} 330 \mathrm{~nm}\right)\end{array}$ & $\begin{array}{l}k_{\mathrm{bc} 2} \times 10^{-2} / \mathrm{s}^{-1} \\
\left(\lambda_{\mathrm{obs}} 330 \mathrm{~nm}\right)\end{array}$ \\
\hline 1.00 & $\mathrm{HCl} 0.100 \mathrm{M}$ & & & $1.7 \pm 0.1$ & \\
\hline 1.30 & $\mathrm{HCl} 0.050 \mathrm{M}$ & & & & \\
\hline 2.00 & $\mathrm{HCl} 0.010 \mathrm{M}$ & $157^{b)}$ & & $1.25 \pm 0.25$ & $1.7 \pm 0.3$ \\
\hline 2.30 & $\mathrm{HCl} 0.0050 \mathrm{M}$ & & & & \\
\hline 2.70 & $\mathrm{HCl} 2.0 \times 10^{-3} \mathrm{M}$ & $72.5 \pm 1^{b)}$ & & $2.2 \pm 0.2$ & $2.1 \pm 0.1$ \\
\hline 3.30 & $\mathrm{HCl} 5.0 \times 10^{-4} \mathrm{M}$ & & & & \\
\hline 3.70 & $\mathrm{HCl} 2.0 \times 10^{-4} \mathrm{M}$ & & & & \\
\hline 3.87 & Acetate $0.025 \mathrm{M} / 0.005 \mathrm{M}$ & 12.6 & $53 \pm 2$ & $4.8 \pm 0.2$ & 5.9 \\
\hline 4.57 & Acetate $0.010 \mathrm{M} / 0.010 \mathrm{M}$ & 4.90 & 15 & 7.3 & \\
\hline 5.27 & Acetate $0.0050 \mathrm{M} / 0.025 \mathrm{M}$ & 1.09 & $2.8 \pm 0.2$ & 8.0 & \\
\hline 6.08 & Phosphate $0.025 \mathrm{M} / 0.005 \mathrm{M}$ & 0.260 & 0.54 & $3.35 \pm 0.1$ & $2.7 \pm 0.25$ \\
\hline 6.76 & Phosphate $0.010 \mathrm{M} / 0.010 \mathrm{M}$ & 0.0122 & 0.036 & & \\
\hline 7.26 & Phosphate $0.005 \mathrm{M} / 0.015 \mathrm{M}$ & 0.0020 & 0.0073 & & \\
\hline 8.12 & TRIS $0.010 \mathrm{M} / 0.010 \mathrm{M}$ & 0.00030 & 0.00054 & & \\
\hline
\end{tabular}

a) Symbols as in ref. 1; rate constants in buffers were not extrapolated to zero buffer concentration, hence, an accurate $\mathrm{pH}-$ rate profile cannot be drawn. ${ }^{b)}$ Single exponential decay.

Il'ichev, Y. V.; Schwörer, M. A.; Wirz. J. J. Am. Chem. Soc., 2004, 126, 4581-4595. 
- 2 -

Table S2: Average Rate Constants $k_{1}$ and $k_{2}$ and their Standard Deviations for the Appearance of 2 and Ratio of the Amplitudes at 236 nm, $A\left(k_{1}\right) / A\left(k_{2}\right)$, in Aqueous Solution.

\begin{tabular}{|c|c|c|c|c|c|}
\hline $\mathrm{pH}$ & {$[\mathrm{HB}] /[\mathrm{B}]$} & $\begin{array}{l}k_{1} \times 10^{2} / \mathrm{s}^{-1} \\
\left(\lambda_{\mathrm{obs}} 360 \mathrm{~nm}\right)\end{array}$ & $\begin{array}{l}k_{1} \times 10^{2} / \mathrm{s}^{-1} \\
\left(\lambda_{\mathrm{obs}} 236 \mathrm{~nm}\right)\end{array}$ & $\begin{array}{l}k_{2} \times 10^{3} / \mathrm{s}^{-1} \\
\left(\lambda_{\mathrm{obs}} 236 \mathrm{~nm}\right)\end{array}$ & $A\left(k_{1}\right) / A\left(k_{2}\right)$ \\
\hline 1.00 & $\mathrm{HCl} 0.100 \mathrm{M}$ & $79 \pm 5$ & & $10 \pm 1$ & 0.587 \\
\hline 1.30 & $\mathrm{HCl} 0.050 \mathrm{M}$ & $38 \pm 4$ & & 8.0 & 0.351 \\
\hline 2.00 & $\mathrm{HCl} 0.010 \mathrm{M}$ & $8 \pm 1$ & $9.4 \pm 2$ & $7.5 \pm 1$ & 0.149 \\
\hline 2.30 & $\mathrm{HCl} 0.0050 \mathrm{M}$ & & 7.2 & 6.5 & 0.075 \\
\hline 2.70 & $\mathrm{HCl} 2.0 \times 10^{-3}$ & & 7.0 & $5.9 \pm 1$ & 0.020 \\
\hline 3.30 & $\mathrm{HCl} 5.0 \times 10^{-4}$ & & & $5.7 \pm 0.2$ & \\
\hline 3.70 & $\mathrm{HCl} 2.0 \times 10^{-4}$ & & & $5.8 \pm 0.2$ & \\
\hline 3.87 & Acetate $0.025 / 0.005$ & & $6.9 \pm 0.3$ & $5.2 \pm 0.2$ & 0.124 \\
\hline 4.57 & Acetate $0.010 / 0.010$ & & $7.4 \pm 1$ & $5.6 \pm 0.2$ & 0.190 \\
\hline 5.27 & Acetate $0.0050 / 0.025$ & 9.5 & $11 \pm 3$ & $5.6 \pm 0.5$ & 0.429 \\
\hline 6.08 & Phosphate $0.025 / 0.005$ & $13 \pm 2$ & $15.5 \pm 2.5$ & $5.0 \pm 0.5$ & 0.587 \\
\hline 6.76 & Phosphate $0.010 / 0.010$ & $27 \pm 5$ & 21 & $5.7 \pm 0.2$ & 0.852 \\
\hline 7.26 & Phosphate $0.005 / 0.015$ & $42.5 \pm 5$ & & 5.5 & \\
\hline
\end{tabular}

The catalytic coefficients determined by fitting of the function $k_{\mathrm{obs}}=k_{0}+k_{\mathrm{H}}\left[\mathrm{H}^{+}\right]+k_{\mathrm{OH}} K_{\mathrm{w}} /\left[\mathrm{H}^{+}\right]$with $K_{\mathrm{w}}=1.59 \times 10^{-14} \mathrm{M}^{2}$ were $k_{0}=(7.1 \pm 0.9) \times 10^{-2} \mathrm{~s}^{-1}$, $k_{\mathrm{H}}=(5.6 \pm 1.2) \mathrm{M}^{-1} \mathrm{~s}^{-1}$, and $k_{\mathrm{OH}}=(1.6 \pm 0.4) \times 10^{6} \mathrm{M}^{-1} \mathrm{~s}^{-1}$ for reaction $\mathbf{C} \rightarrow \boldsymbol{2}\left(k_{1}\right)$ and $k_{0}=(5.7 \pm 1.5) \times 10^{-3} \mathrm{~s}^{-1}$ and $k_{\mathrm{H}}=(4.7 \pm 0.8) \times 10^{-2} \mathrm{M}^{-1} \mathrm{~s}^{-1}$, with $k_{\mathrm{OH}}$ set to zero for reaction $\mathbf{3} \rightarrow \mathbf{2}\left(k_{2}\right)$.

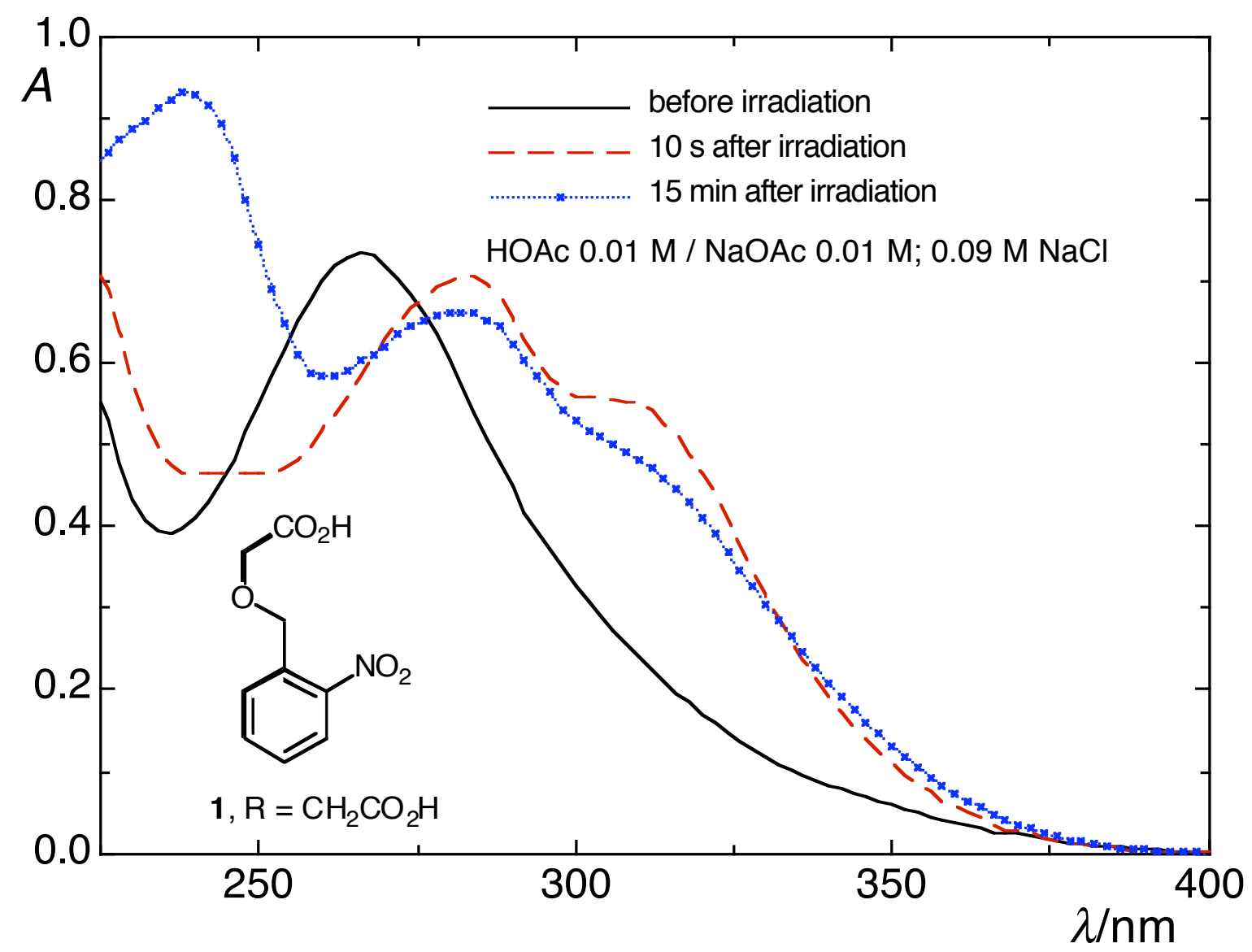

Figure S1. Absorbance $A$ of a $1.3 \times 10^{-4} \mathrm{M}$ solution of $\mathbf{1}, \mathrm{R}=\mathrm{CH}_{2} \mathrm{CO}_{2} \mathrm{H}$, in aqueous acetate buffer before (- - ), immediately after (-- ), and 15 min after $(\cdots \times \cdots \times \cdots)$ irradiation by 10 laser flashes at $308 \mathrm{~nm}$. The second spectrum $(---)$ is attributed to a mixture of $\mathbf{C}\left(\mathrm{R}=\mathrm{CH}_{2} \mathrm{CO}_{2} \mathrm{H}\right)$ and $\mathbf{3}$, the third to the final photoproduct 2 . 
- 3 -

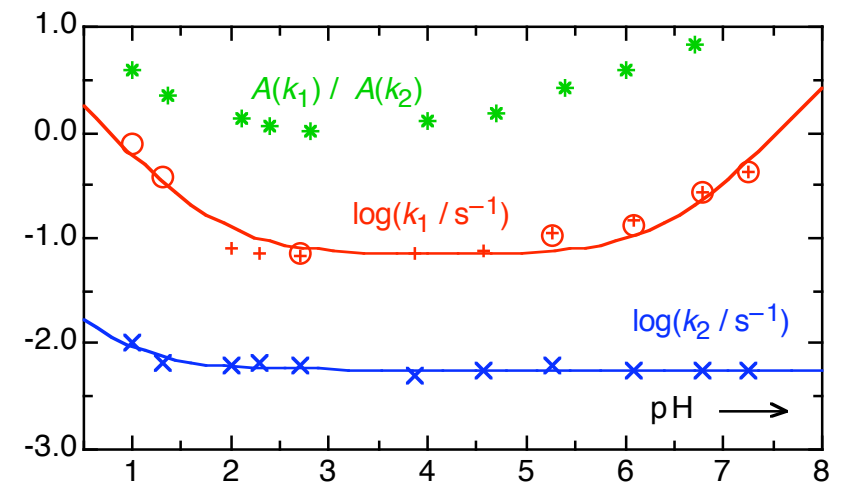

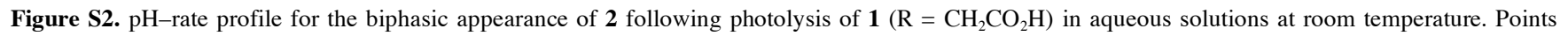
marked + and $\times$ were measured at $236 \mathrm{~nm}$ (conventional spectrometer, growth), points marked $\mathrm{O}$ at $360 \mathrm{~nm}$ (flash photolysis, decay). The points marked $*$ show the ratio of the amplitudes of the two growth components. A function allowing for acid and base catalysis was fitted to the rate data. The term for base catalysis was set to zero for fitting of $k_{2}$. Outside the $\mathrm{pH}$-range of $2-6, k_{1}$ had to be measured by flash photolysis. The rate data obtained by the two methods agreed well within $2<\mathrm{pH}<6$. The data points are taken from Tables S1 and S1. 